\title{
Changes in Residential Structure in 20th-Century Kyoto City
}

KIRIMURA Takashi

\section{Introduction}

II Classification Based on Residential Characteristics of the Three Time Points

(1) Statistical Materials

(2) Data on Residential Characteristics

(3) Classification Procedures

\section{Changes in Residential Structure}

(1) Residential Structure in 1911

(2) Residential Structure in 1965

(3) Residential Structure in 2005

\section{Conclusion}

Key Words : residential structure, intra-urban structure model, Kyoto City, Self-Organizing Map (SOM)

\section{Introduction}

How has the residential structure in large cities of Japan changed from modern to contemporary times? While not many geographical studies have been done from a long-term perspective, which can answer this question, there are some fragmentary studies that are useful. For Osaka from early modern to modern times, the process of how its inner area was formed, along with changes in the labor market due to industrialization, has been revealed. On the other hand, for Tokyo in the 1920s, when it was undergoing a transition from early modern to modern times, it has been shown that the residential structure of post-modern times still remained. This was because the residential structure of Tokyo was formed in such a way that new urban structures formed after the Meiji period were added to the urban structure of the Edo period, or by improving the preexisting urban structure. Later in the 1970s, Japanese cities, which had undergone damage from World War II and then high economic growth, were often taken up as a subject in research on residential structure, which centered on a factorial ecology approach. In Tokyo of 1970s, there existed a sectoral structure based on occupation and an urban residential structure of a lifestyle-based concentric type, on which interpretations have been made based on the conventional framework used to describe the urban structure of Tokyo. After the 1980s, centering on the three major metropolitan areas in Japan, the suburbanization of workplaces accelerated and the number of workers in tertiary industries increased. City centers faced problems of population decline and aging. While there was an increase in population again in city centers after the latter half of the 1990s, Japanese geographers did not pay much attention to residential structure after that. In past research on the residential structure of large cities in 
Japan, the findings were interpreted based on the historical urban structure of early modern times in the beginning of the modern period, whereas in the 1970s interpretations were made based on the classical intra-urban structure model. However, since the research was rarely conducted after that.

While research based on a long-term perspective had long been needed, most of past research on the residential structure targeted a single time point. The lack of a long-term perspective in the interpretations of residential structures involves the risk of interpreting the results based on the social context of the time when the research was conducted. On the other hand, long-term analyses pose two challenges. One is that materials are limited in availability and the other is a clear discontinuity in urban structure due to the damage caused by World War II. In order to reveal changes in the residential structure of large cities in Japan after early modern times, therefore, analyses should not only be made based on a long-term and historical perspective, but also cities that have many materials available and little discontinuity in their urban structure due to war damage should be found and targeted.

Kyoto City, the target city of this paper, has not experienced a significant disaster or war damage that could have changed its urban structure altogether after the modern period. Therefore, Kyoto City is an appropriate target for the elucidation of long-term changes in residential structure. Moreover, for material availability, the report of the extraordinary regional census conducted in 1911 is stored in the National Diet Library. The report provides us with data on a variety of residential characteristics according to the 1,695 towns that comprised Kyoto City at the time. For detailed small area statistics before this census, the Shumon Aratamecho and Nimbetsucho (both are family registers created in the Edo period) are often used in historical demography. However, due to their nature as historical materials, it is difficult to obtain information by city. For these reasons, the year 1911 was set as the starting point of this paper.

The purpose of this study is to reveal the changes that took place in the residential structure of Kyoto City over about 100 years from 1911 up to the present. The following three time points were set as target time cross sections in view of availability of small area statistics on residents : 1911, 1965 and 2005. The year 1911 is when the extraordinary census was conducted and the years 1965 and 2005 are when the national population census was carried out. The national population census conducted in 2005 is the latest small area statistics available, whereas the small area statistics of 1965 is the oldest material available after World War II, which provides detailed tabulation categories by small area unit. Thus, each of the three time points, which were selected mainly for material availability reasons, also has some historically significant background. First of all, the year 1911 was when the following three major projects were about to be completed: the construction of the second Biwako canal, upgrading of waterworks and road expansion. The second canal and waterworks were completed in 1912 and the road expansion project was completed in the following year in 1913. Upon the completion of the three major projects, waterworks were installed and began supplying water, which was expected to improve the city's hygienic environment and Kyoto City's streetcar service began operations on the expanded roads, which was expected to improve intra-urban transportation. The administrative district of Kyoto City at the time included only the area centering on old Kyoto (Figure 1). The year 1965 was when the economy was growing rapidly and urbanization accelerated in the surrounding areas of Kyoto City. Compared with 1911, the administrative district expanded greatly due to municipal mergers and infrastructure development in the city 


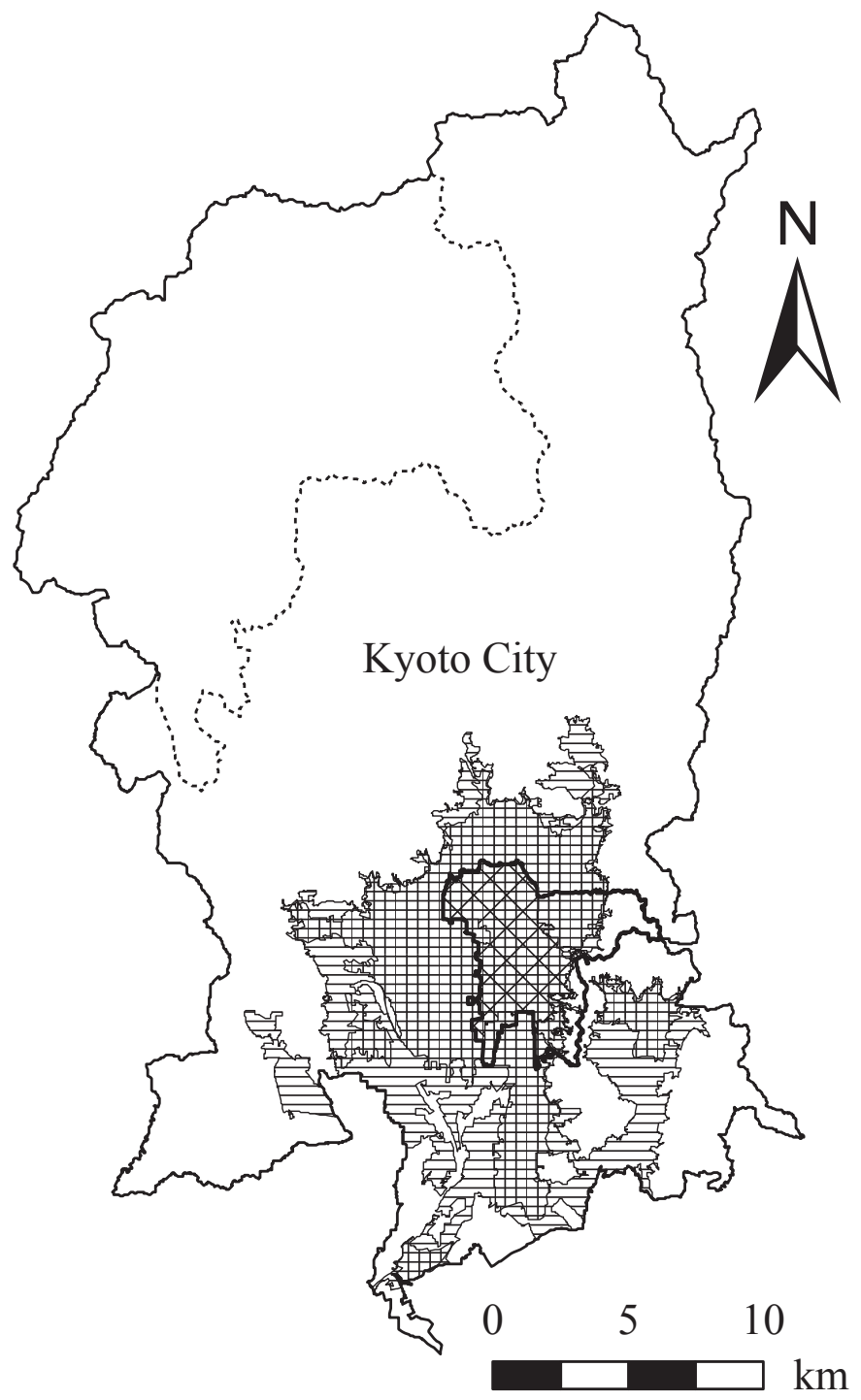

\section{Legends}

- Administrative boundary in 1911

...... Administrative boundary in 1965

— Administrative boundary in 2005

Urbanized area in the 1910s

पा] Urbanized area in 1965

Urbanized area in 2005

Figure 1. Administrative areas and urbanized areas of Kyoto City in 1911, 1965 and 2005.

The urbanized areas in 1965 and 2005 are the Density Inhabited Districts (DIDs). DID is composed of a group of contiguous Basic Unit Blocks each of which has a population density of about 4,000 inhabitants or more per square kilometer and whose total population exceeds 5,000. The urbanized areas in the 1910s are shown in topographical maps in the 1910s. 
accelerated, such as the implementation of a land readjustment project in the areas surrounding the old city. In 2005, which can be viewed as the present, the only change in terms of the administrative district was the incorporation of Keihoku town, which was located in the northern part of Kyoto. However, the urban area of Kyoto City expanded greatly due to the development of new towns to meet the demands of increased population and the further acceleration of urbanization. Thus, since the administrative districts of Kyoto City at the three time points differ from one another, due care should be taken when comparing them.

In the next chapter, the outline of the resident characteristic data used in this study and the method used to classify the residential characteristics into types, which will be used to identify the residential structure, are discussed. Regarding the comparison of the residential structures among the three time points, since data at the three time points differ in categories and the size of the target area due to the restricted availability of materials and therefore they cannot be compared directly, it was decided to make relative comparisons between two time points. Then, a typological analysis, which is useful for this type of relative comparison, was conducted. For the three time points, variables were set separately to represent the residential characteristics of each time point to conduct analysis by classifying them into types. The spatial distributions of the types thus obtained were reviewed via special autocorrelation scale. By comparing the results of this review with the findings of past research on the urban structure of Kyoto City after the early modern period, particularly after the last Edo period, the paper attempts to reveal the changes in the residential structure of Kyoto City.

\section{Classification Based on Residential Characteristics of the Three Time Points}

\section{(1) Statistical Materials}

In 1911, an extraordinary regional census was conducted by Kyoto City with the aim of obtaining accurate population information, which family registers or the former resident registers could not provide, targeting residents living in Kyoto City as of 12:00 a. m., November 1, 1911. The census was implemented under the leadership of the Director-General of the Cabinet Bureau of Statistics in view of a future national population census, which had not yet been conducted in Japan. While surveys similar to the extraordinary regional census had been implemented in Taiwan, Tokyo and Kobe, it was the first census for Kyoto City. In light of the fact that it was carried out by policemen and influential people in each town in the city, the author deems the tabulated figures accurate. Among the statistical tables created after the census, five tables on the following categories were tabulated by town : household type, household size, age group, religion and occupation.

For the results of the 1965 national population census, the statistical tables by town in the "Kyoto-shi no jinko (Population of Kyoto City)," which was published by Kyoto City based on the results of the 1965 national population census, are used. However, since the spatial unit in these statistical tables by town were created based on enumeration districts, the border of the district does not necessarily correspond to that of the towns that actually existed at the time. Nevertheless, in light of the rarity of the material, the tables in the source are used for analysis in this paper by deeming that all districts correspond to towns. However, since, in these tables, population is tabulated by age group, employment status, industry and occupation and there is no information on household, the household figures tabulated independently by Kyoto City for "Kyoto-shi no chobetsu jinko (Population by Town of Kyoto City)" based on the preliminary figures of 
the 1965 National Population Census are used by re-tabulating them by town in the "Kyoto shi no jinko."

For the results of the 2005 national population census, the small area tabulation results created and published by the Statistics Bureau are used. However, since information on towns with an extremely small population requires confidential treatment, these towns were combined and used. For tabulation categories, while the number decreased drastically compared to that of the 2000 census, it still contains a variety of categories, such as age group, housing, household type, industry and occupation.

\section{(2) Data on Residential Characteristics}

Regional classification was conducted separately for each of the three time points. In an effort to facilitate a relative comparison of the results, the variables, which are used at each time point to represent residential characteristics, were selected in such a way that they resemble one another in meaning as much as possible. Naturally, the results of the 1965 national population census, which has the least number of tabulation categories, were adopted as the criteria for selecting the variables.

For the year 1965, among the number of workers by industry and by occupation, both of which are similar in nature, the number of workers by occupation is used. For variables, those shown in Table 1, including population density, sex, age group, employment status and occupation, are used. For the year 2005, more detailed tabulation categories were used in the 2005 census than in the 1965 census. However, for the sake of convenience of comparison, the same variables as those selected for 1965 are used in this paper.

For the year 1911, the tabulation categories differ from those of the two other time points. The occupational categories in the extraordinary regional census are based on those used in the regional census of Tokyo, where population by occupation is tabulated separately under the two categories of 'occupied persons' and 'dependents'. Dependents include non-working family members and the occupations in the dependent category are represented by the occupation of the supporter or husband. Since the 'occupied persons' in the extraordinary regional census can be said to correspond to 'employed persons' in the 1965 and 2005 National Population Census, the variables for the number of workers by occupation are adopted.

The values of 19 variables for each of the three time points thus created are standardized by variable. This is to avoid the impact of difference in value scale when classifying them.

\section{(3) Classification Procedures}

In past research on residential structure, a method combining factor analysis and cluster analysis has been used for the classification of residential characteristics. However, this method has a drawback due to the technical limitations of factor analysis in that if there are not enough variables of the same type, it is difficult to extract factors related to a particular characteristic. Therefore, it is not the best method for this research, where the number of available variables is limited. This is why the Self-Organizing Map ( $\mathrm{SOM}$ ) method is adopted for classification in this paper. SOM is an algorithm modeled after the mechanism of self organization through learning by the brain neurons of organisms. Figure 2 illustrates the basic SOM structure. By applying the algorithm to residential characteristics and having the "neurons" learn the characteristics in a repeated manner, the residential characteristics can be classified into types. Although SOM is not a common classification method and there are not many geographical studies that use SOM 
Table 1. Variables of residential characteristics in 1911, 1965 and 2005.

\begin{tabular}{|c|c|c|c|}
\hline Year & No & Name & Description \\
\hline \multirow{19}{*}{1911} & 1 & POPDENS & population density (the number of people per sq $\mathrm{km}$ ) \\
\hline & 2 & MALE & ratio of male population \\
\hline & 3 & FAMALE & ratio of female population \\
\hline & 4 & HHSIZE & average value of household member \\
\hline & 5 & AGE01_10 & ratio of resident population aged $1-10$ years \\
\hline & 6 & AGE11_20 & ratio of resident population aged $11-20$ years \\
\hline & 7 & AGE21_30 & ratio of resident population aged 2130 years \\
\hline & 8 & AGE31_40 & ratio of resident population aged $31-40$ years \\
\hline & 9 & AGE41_50 & ratio of resident population aged $41-50$ years \\
\hline & 10 & AGE51_60 & ratio of resident population aged $51-60$ years \\
\hline & 11 & AGE61_70 & ratio of resident population aged $61-70$ years \\
\hline & 12 & AGE71_ & ratio of resident population aged 71 or more years \\
\hline & 13 & OCСРОР & ratio of occupied population \\
\hline & 14 & AGRW & ratio of agricultural workers \\
\hline & 15 & INDW & ratio of industrial workers \\
\hline & 16 & COMW & ratio of commercial / traffic service workers \\
\hline & 17 & PROW & ratio of civil servants / professional workers \\
\hline & 18 & OTHW & ratio of other workers \\
\hline & 19 & UNEMP & ratio of unemployed people \\
\hline \multirow{19}{*}{$\begin{array}{c}1965 \\
\& \\
2005\end{array}$} & 1 & POPDENS & population density (the number of people per sq $\mathrm{km}$ ) \\
\hline & 2 & MALE & ratio of male population \\
\hline & 3 & FAMALE & ratio of female population \\
\hline & 4 & HHSIZE & average value of household members \\
\hline & 5 & AGE00_14 & ratio of resident population aged $0-14$ years \\
\hline & 6 & AGE15_24 & ratio of resident population aged $15-24$ years \\
\hline & 7 & AGE25_29 & ratio of resident population aged $25-29$ years \\
\hline & 8 & AGE30_39 & ratio of resident population aged $30-39$ years \\
\hline & 9 & AGE40_49 & ratio of resident population aged $40-49$ years \\
\hline & 10 & AGE50_59 & ratio of resident population aged $50-59$ years \\
\hline & 11 & AGE60_69 & ratio of resident population aged $60^{-69}$ years \\
\hline & 12 & AGE70_ & ratio of resident population aged 70 or more years \\
\hline & 13 & OCCPOP & ratio of occupied population \\
\hline & 14 & EMP & ratio of employees \\
\hline & 15 & SELFEMP & ratio of self-employed workers \\
\hline & 16 & AGRW & ratio of agricultural / forestry workers \\
\hline & 17 & BLUEW & ratio of blue-collar workers \\
\hline & 18 & GRAYW & ratio of gray-collar workers \\
\hline & 19 & WHITW & ratio of white-collar workers \\
\hline
\end{tabular}

as a classification method, it has been found that this method can overcome the aforementioned drawback of factor analysis. For detailed procedures of classification via SOM, please refer to Nakaya and Openshaw et ${ }^{24}$ al.

Below is the summary of the characteristics of classification via SOM. First of all, neurons, which eventually become individual types, are arranged on the output layer. In SOM, the figure, which shows the characteristics of each type on the output layer, is called a 'map'. The map plays a very important role not only in classification, but also in analysis via SOM. The characteristics of each type can be understood by the weights corresponding to the variables in the original data. However, it is normally achieved by creating a map for each variable that shows the weight value of each type. These variable maps are collectively called a 'component plane'. Another characteristic of classification via SOM is that the closer the location of the types on the map, 


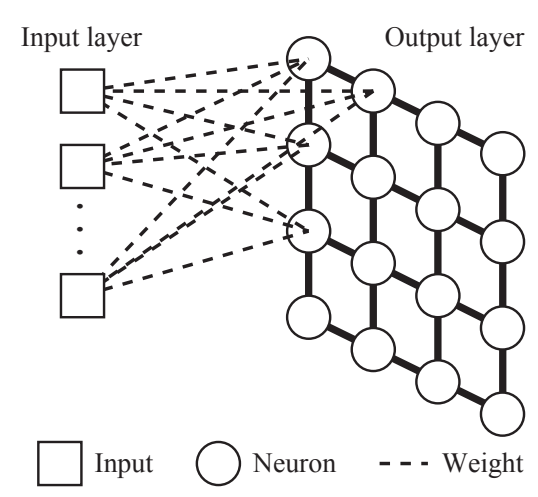

Figure 2. Structure of basically SOM.

the greater the similarity in characteristics. The formation of this relationship is due to the fact that when individual neurons learn the characteristics of the original data, the neighboring neurons learn similar characteristics. The neighboring range must be set in such a way that it is great at an early stage of repeated learning and becomes zero at the end. This characteristic of "similar types in the neighboring area" enables the examination of various characteristics of each type by using the SOM map, just like an ordinary map. While it is necessary to have a large number of types in order to fully use a map with the aforementioned characteristics, having too many types makes it difficult to interpret individual types or examine their spatial distributions. Therefore, it is necessary to regroup the types obtained via SOM to create groups of fewer types by using a method such as Ward's method. These groups of types are called "neuron types" in this paper.

When using SOM, some parameters must be set. For convenience of comparison, common parameters were set for each time point as follows. For the number of types, ten neurons were placed vertically and another ten horizontally on the output layer to enable a map-based examination, creating a total of 100 types. These types were then grouped via Ward's method into several neuron types. The initial neighboring range was set at three in light of the placement of neurons. The frequency of learning repetition was set at 50,000 since the largest number of districts to be learned is 4,932 (in 2005). These are the parameters required for a normal SOM. Since the analysis is conducted using small area statistics in this paper, an SOM improved to fit the characteristics of small area statistics is used. This improved SOM is hereinafter referred to simply as SOM. In this SOM, the range for the update of weight values is restricted to minimize the impact of outliers, which often appear in small area statistics, when updating weight values during the stage of learning the characteristics of the original data. In light of the fact that the value of each variable has been standardized, the minimum and maximum values of this parameter were set at -0.3 and 0.3 respectively.

\section{Changes in Residential Structure}

\section{(1) Residential Structure in 1911}

First of all, SOM was applied to residential characteristics of the 1,695 districts as of 1911 and nine neurons types were obtained. Individual neuron types were named mainly according to the dominant occupation type and the household size, while taking the characteristics of each neuron type into consideration (Table 2). Neuron type MN1 was named "Commercial Worker and Large Household," MN2 "Commercial Worker and Medium Household," MN3 "Small Population," MN4 "Industrial Worker and Large Household," MN5 "Industrial Worker and Small Household," MN6 "Female Commercial Worker and Large Household," MN7 "Other Occupation and Small Household," MN8 "Civil Servant / Professional Worker and Small Household" and MN 9 "Civil Servant / Professional Worker and Large Household."

At the time of 1911, the urban area centering on Kyoto corresponded almost perfectly to the administrative area of Kyoto City. The development of the suburban residential area had not 
Table 2. Characteristics of neuron types in 1911 (average values of neurons' weights).

\begin{tabular}{c|c|r|r|r|r|r|r|r|r|r}
\hline No & Variable name & \multicolumn{1}{c|}{ MN1 } & \multicolumn{1}{c|}{ MN2 } & MN3 & \multicolumn{1}{c}{ MN4 } & MN5 & MN6 & MN7 & MN8 & MN9 \\
\hline 1 & POPDENS & -0.31 & 0.08 & -1.12 & -0.10 & -0.20 & 0.30 & 0.39 & -0.63 & -1.20 \\
2 & MALE & 1.32 & 0.35 & -2.84 & 0.02 & -0.01 & -1.84 & 0.00 & -0.34 & 1.01 \\
3 & FAMALE & -1.27 & -0.33 & -3.23 & -0.05 & 0.07 & 1.90 & 0.05 & 0.40 & -0.96 \\
4 & HHSIZE & 2.29 & 0.29 & -1.52 & 0.58 & -0.35 & 0.61 & -0.49 & -0.32 & 0.27 \\
5 & AGE01_10 & -1.19 & -0.43 & -2.08 & -0.21 & 0.63 & -1.20 & 0.25 & 0.03 & -1.33 \\
6 & AGE11_20 & 2.08 & 0.47 & -1.38 & 0.96 & -0.45 & 0.13 & -0.55 & -0.40 & -0.07 \\
7 & AGE21_30 & 0.46 & 0.20 & -2.05 & -0.20 & -0.14 & 1.23 & -0.18 & -0.03 & 0.89 \\
8 & AGE31_40 & -1.12 & -0.28 & -2.13 & -0.47 & 0.28 & -0.11 & 0.33 & 0.17 & -0.34 \\
9 & AGE41_50 & -1.08 & -0.15 & -1.95 & -0.51 & 0.05 & 0.10 & 0.51 & 0.05 & -0.49 \\
10 & AGE51_60 & -0.84 & -0.18 & -1.73 & -0.52 & 0.16 & 0.01 & 0.35 & 0.35 & 0.24 \\
11 & AGE61_70 & -0.91 & -0.13 & -1.40 & -0.42 & 0.03 & -0.17 & 0.37 & 0.34 & 0.27 \\
12 & AGE71_ & -0.67 & -0.03 & -0.94 & -0.22 & -0.22 & -0.06 & 0.09 & 0.97 & 0.02 \\
13 & OCCPOP & 0.52 & 0.04 & -3.07 & 0.32 & 0.06 & 1.25 & -0.32 & -0.35 & 1.16 \\
14 & AGRW & -0.25 & -0.06 & -0.26 & -0.20 & 0.32 & -0.05 & -0.05 & -0.06 & 0.19 \\
15 & INDW & -1.14 & -0.66 & -1.21 & 1.22 & 0.75 & -1.40 & -0.13 & -0.42 & -1.29 \\
16 & COMW & 1.97 & 1.00 & -0.69 & -0.82 & -0.59 & 0.97 & 0.05 & -0.47 & -0.87 \\
17 & PROW & -0.63 & -0.17 & -0.59 & -0.48 & -0.26 & 0.58 & 0.03 & 0.94 & 2.31 \\
18 & OTHW & -0.58 & -0.41 & -0.58 & -0.49 & -0.08 & 1.44 & 0.36 & 0.20 & -0.19 \\
19 & UNEMP & -0.40 & -0.12 & -0.51 & -0.43 & -0.35 & -0.15 & 0.02 & 1.19 & 2.79 \\
\hline
\end{tabular}

started in full swing yet and it seemed that most of the residents lived in the same area as or near their workplaces. If each neuron type is interpreted based on this assumption, then it can be said that the occupation composition of the residents almost matches with the composition of business establishments. Two neuron types of MN1 and MN2, which comprise many residents working in the field of commerce or traffic service, show a distribution pattern along major roads, suggesting that these areas along main roads may be major commercial or business districts (Figure 3). Particularly, the area where the MN1 type concentrates can be viewed as a city center of the time. On the other hand, the MN6 type, which also comprises many residents who work in the commercial or transport service field, shows an aggregated distribution centering on the districts where red-light areas are located, suggesting that the areas where this type spreads are entertainment districts. Among the two neuron types where many residents work in the industrial field, the MN4 type concentrates in the north central part of Kamigyo-ku (ward) and the northwestern part of Shimogyo-ku, whereas the MN5 type spreads in the area surrounding the MN4 centering on Kamigyo-ku and seems to form a textile industry area of 'Nishijin.' The MN8 and MN9 types, where most residents are professional workers or civil servants, spread mainly in the northeastern part of Kamigyo-ku. However, the districts, in which large temples, shrines, universities, or public facilities are located, also belong to these neuron types. The MN7 type, where residents' occupations are evenly spread among various fields, with slightly more residents working in 'other fields', centers on Shimogyo-ku and spreads as if to fill niches among other neuron types. Other occupations mainly include day workers, licensed prostitutes and office workers. Assuming that the licensed prostitutes concentrate on the MN6 and its surrounding areas, it can be said that most of the MN7 type are office workers and daily workers.

Viewed in terms of the nine neuron types, how has the residential structure at the time of 1911 in the early modern period inherited the residential structure of the early modern period ? As a lead to find the answer, the results of historical demographic analysis conducted by Hamano on 


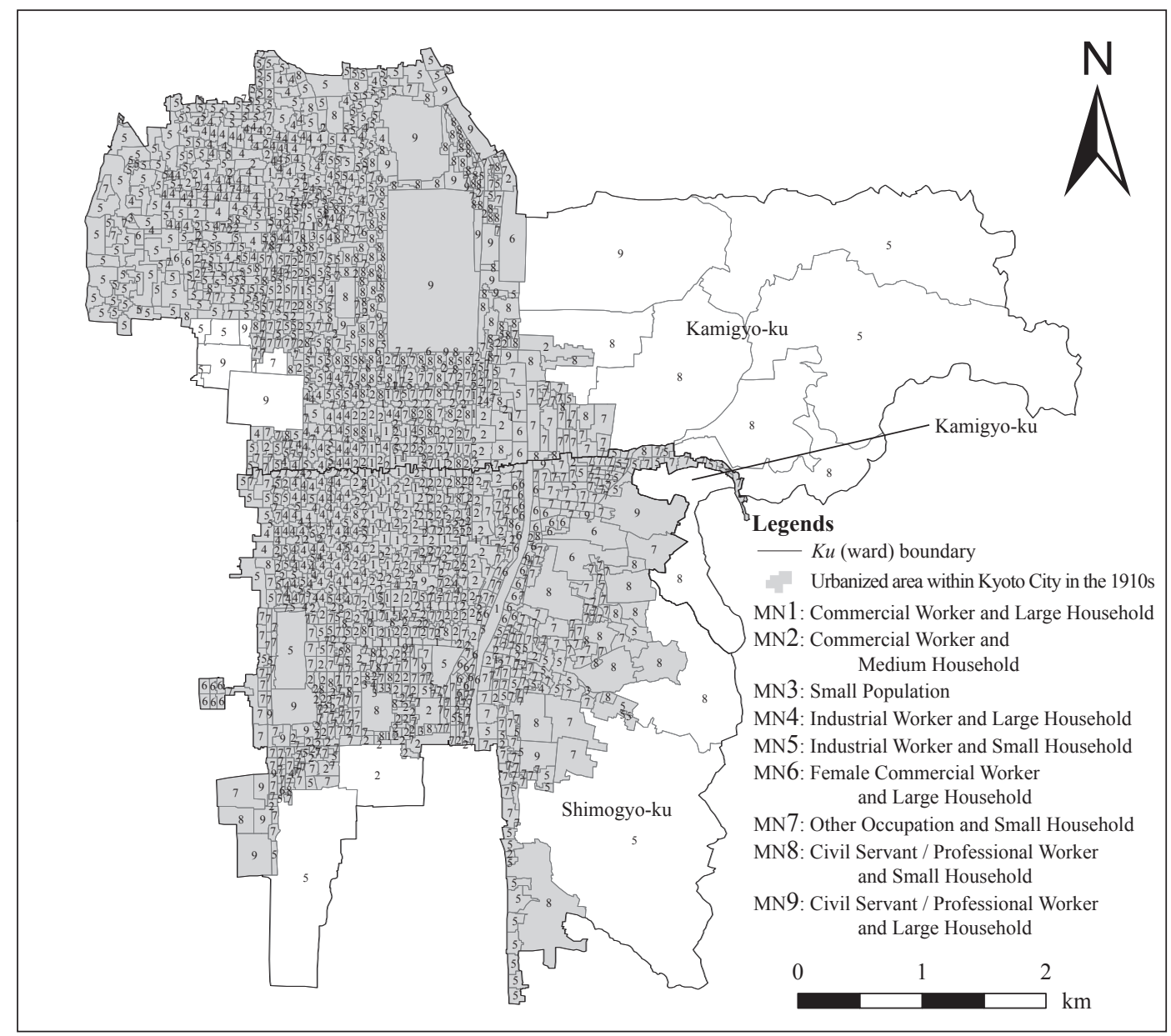

Figure 3. Distributions of neuron types in 1911.

The number shows that of neuron type.

some towns in Kyoto in the late Edo period are of great use. The neuron types, such as MN1, MN2 and MN4, which concentrate on the centers of Kamigyo-ku and Shimogyo-ku and where the household size is large and the ratio of population aged 11 to 20 is high, can be viewed as a remnant from the late Edo period when a large number of 'hokonin (apprentices) ' lived in the city center. While there are more men in the MN1 type that spreads prominently in Shimogyo-ku, there is not much difference in the number of men and that of women in the MN4 type in the western part of Kamigyo-ku. This fact seems to reflect the succession of contrasting conditions of the late Edo period when there were many merchants with male hokonin in Shimogyo-ku, whereas there were many female hokonin working in textile-related business in Kamigyo-ku. Moreover, the characteristics of the MN7 type, except office workers, correspond to the people in the so-called urban miscellaneous group. Shimizu-cho in Shimogo-ku, an example of the group cited by Hamano, belongs to this neuron type. The MN7 type spreads not only in the margin of the entire Kyoto City, but also around the boundaries of Kamigyo-ku and Shimogyo$\mathrm{ku}$, suggesting that the residential area of the urban miscellaneous group spreads in the marginal area of each urban core district. On the other hand, the MN8 and MN9 types are comprised of 
the combination of post-modern factors, such as universities and public institutions and religious forces, such as large temples and shrines. It is difficult, however, to compare these two types with the results of research conducted before modern times. It can be said, however, that at least these large temples and shrines are a factor that also existed at the late Edo period.

The residential structure of 1911, which features the residential structure inherited from the late Edo period and the addition of new factors of modern times, can be described as follows. The household size tended to be large in the central areas of Kamigyo-ku and Shimogyo-ku, where there were many young hokonin and become smaller at the margin, forming a concentric pattern. Kamigyo-ku is a textile industry-based district centering on Nishijin, most of which is occupied by the industrial worker-dominant neuron types. On the other hand, in Shimogyo-ku, a female-dominant entertainment area spreads in the eastern part and the industrial workerdominant neuron type area in the western part, as if to surround the urban commercial workerdominant district in a sectoral manner. In the surrounding region of these two areas spreads a neuron type comprising a mix of workers in the urban miscellaneous group and office workers. On the other hand, when the entire city area of Kyoto of the time is examined, there are major accumulations of civil servants and professional workers in the northeastern part, that is, in the eastern part of Kamigyo-ku. These are the residential areas where the traditional elite centering on large temples and shrines and occupational groups of civil servants and teachers, which were formed after the Meiji period, live. These residential areas seem to have positioning different from the structure centering on the city center, which existed in Kamigyo-ku and Shimogyo-ku. Thus, the residential structure of Kyoto in 1911 showed a combination of the residential structure inherited from the late Edo period and the new elements added after the Meiji period, most of which corresponds to the conditions of Tokyo City in 1920. However, the new elements introduced after the Meiji period are not distributed across the city as in the case of Tokyo City, but forms a cluster, which is rather large as a sector, in the northeastern part of the city.

\section{(2) Residential Structure in 1965}

SOM was then applied to the residential characteristics of 1965 and the 2,524 districts were classified into eight neuron types. Each neuron type was named, as in 1911, mainly according to the dominant occupation type and the household size, while taking the characteristics of each neuron type into consideration (Table 3). Neuron type SN1 was named "White-Collar Worker and Small Household," SN2 "Small Population," SN3 "Young Blue-Collar Worker and Medium Household," SN4 "Gray-Collar Worker and Large Household," SN5 "Female Gray-Collar Worker and Medium Household," SN6 "Blue-Collar Worker and Medium Household," SN7 "SelfEmployed Blue-Collar Worker and Medium Household" and SN8 "Agriculture / Forestry Worker and Large Household." For the interpretation of spatial distribution of each neuron type of 1965, it should be noted that some of the districts have areas larger than others.

Compared to 1911, the area of Kyoto City in 1965 expanded about 20 times, from $31.28 \mathrm{~km}^{2}$ to $610.61 \mathrm{~km}^{2}$, due to the incorporation of surrounding municipalities conducted several times. On the other hand, the population was $1,365,007$, about three times the population in 1911 . However, the population of the city area $(463,836)$ and that of the old city area of 1911 were about the same. The system of hokonin, which had played an important role in the population of the city center until the modern period, already became hard to maintain due to the enactment of the Labor Standards Law in 1947. In the surrounding area of the old city of 1911, suburban sprawl accelerated and land readjustment projects and housing land development by private 
Table 3. Characteristics of neuron types in 1965 (average values of neurons' weights).

\begin{tabular}{c|c|r|r|r|r|r|r|r|r}
\hline No & Variable name & \multicolumn{1}{c|}{ SN1 } & \multicolumn{1}{c}{ SN2 } & \multicolumn{1}{c}{ SN3 } & \multicolumn{1}{c}{ SN4 } & \multicolumn{1}{c}{ SN5 } & \multicolumn{1}{c}{ SN6 } & \multicolumn{1}{c}{ SN7 } & \multicolumn{1}{c}{ SN8 } \\
\hline 1 & POPDENS & -0.57 & -1.09 & 0.14 & -0.13 & 0.01 & -0.70 & 0.95 & -1.29 \\
2 & MALE & 0.22 & -3.43 & -0.10 & 0.41 & -0.49 & 0.40 & -0.22 & 0.11 \\
3 & FAMALE & -0.43 & -4.80 & 0.16 & -0.34 & 0.55 & -0.34 & 0.28 & -0.05 \\
4 & HHSIZE & -0.36 & -1.50 & 0.07 & 0.32 & -0.05 & -0.09 & -0.02 & 0.30 \\
5 & AGE00_14 & -0.36 & -2.52 & -0.35 & -1.00 & -0.66 & 0.85 & 0.04 & 0.99 \\
6 & AGE15_24 & 0.24 & -1.76 & 0.42 & 1.21 & 0.00 & -0.43 & -0.22 & -0.69 \\
7 & AGE25_29 & -0.42 & -2.00 & -0.11 & 0.13 & -0.25 & 0.87 & -0.22 & -0.32 \\
8 & AGE30_39 & -0.12 & -2.61 & -0.38 & -0.75 & -0.38 & 0.88 & 0.02 & 0.15 \\
9 & AGE40_49 & 0.19 & -2.58 & -0.15 & -0.13 & 0.39 & -0.31 & 0.17 & 0.10 \\
10 & AGE50_59 & -0.03 & -2.09 & 0.35 & -0.15 & 0.52 & -0.78 & 0.21 & -0.33 \\
11 & AGE60_69 & -0.05 & -1.68 & -0.02 & 0.08 & 0.78 & -0.75 & 0.28 & 0.05 \\
12 & AGE70_ & 0.09 & -1.33 & 0.01 & -0.17 & 0.55 & -0.72 & 0.27 & 0.61 \\
13 & OCCPOP & -1.28 & -4.48 & -0.01 & 1.12 & 0.55 & -0.15 & 0.17 & 0.15 \\
14 & EMP & 0.63 & -2.66 & 0.26 & 0.04 & -1.01 & 0.79 & -0.46 & -1.08 \\
15 & SELFEMP & -0.63 & -1.95 & -0.26 & -0.34 & 0.96 & -0.78 & 0.62 & 0.85 \\
16 & AGRW & -0.18 & -0.24 & -0.23 & -0.29 & -0.28 & 0.10 & -0.24 & 3.22 \\
17 & BLUEW & -0.78 & -1.74 & 0.31 & -1.18 & -1.04 & 0.51 & 0.38 & -0.47 \\
18 & GRAYW & -0.41 & -1.31 & -0.17 & 1.65 & 1.51 & -0.56 & 0.07 & -0.99 \\
19 & WHITW & 1.63 & -0.75 & 0.00 & -0.22 & -0.25 & -0.03 & -0.37 & -0.79 \\
\hline
\end{tabular}

capital were actively implemented after the 1920s, providing high-quality residential areas. Compared to other major cities in Japan, the war damage caused by World War II was not as devastating as to completely change the city structure. In the rural areas that were incorporated into Kyoto City, housing land development accelerated along with the establishment of factories in these areas after the war and the urban area expanded significantly compared to the old city area in 1911. Despite these changes, suburban agriculture was still actively conducted.

The spatial distribution of each neuron type shows that the SN8 type spreads widely in the margin of Kyoto City, where agricultural land use predominates, such as the northern and southern parts of Ukyo-ku, the northern part of Sakyo-ku and the western and eastern parts of Fushimi-ku. The blue-collar-dominant SN6 type, featuring slightly more workers in agriculture and forestry than in other fields, spreads between the SN8 type area and the urban area, such as Ukyo-ku, Minami-ku, Fushimi-ku and Yamashina-ku, suggesting a mix of factories established in the surrounding area and suburban agriculture (Figure 4). The SN6 type features a larger population of those under 15 , in their late 20 s and in their 30 s, which seems to suggest that there are many young families. The four wards of Kamigyo, Nakagyo, Shimogyo and Higashiyama centering on the city area of 1911 are comprised mainly of the following four neuron types: SN3, SN4, SN5 and SN7. While the distribution of the SN4 type, where workers in sales and service industries are prominent, has a remnant of the city center of 1911, which comprised Kamigyo-ku and Shimogyo-ku, the accumulation in the eastern part of Nakagyo-ku seems to be a new formation. Although the SN5 type that spreads heavily on the eastern part of the SN4 type area seems to be a neuron type that corresponds to the MN6 type of 1911, the range of distribution has expanded and it seems to include people in the more general graycollar group. The blue-collar-dominant SN7 type and SN3 type is predominate in the western parts of Kamigyo-ku, Nakagyo-ku and Shimogyo-ku and the southern part of Higashiyama-ku. While the SN7 type spreads centering on the old city of 1911, it also forms a small accumulation in the southern part of Kita-ku. This seems to be due to the expansion of the textile industry 


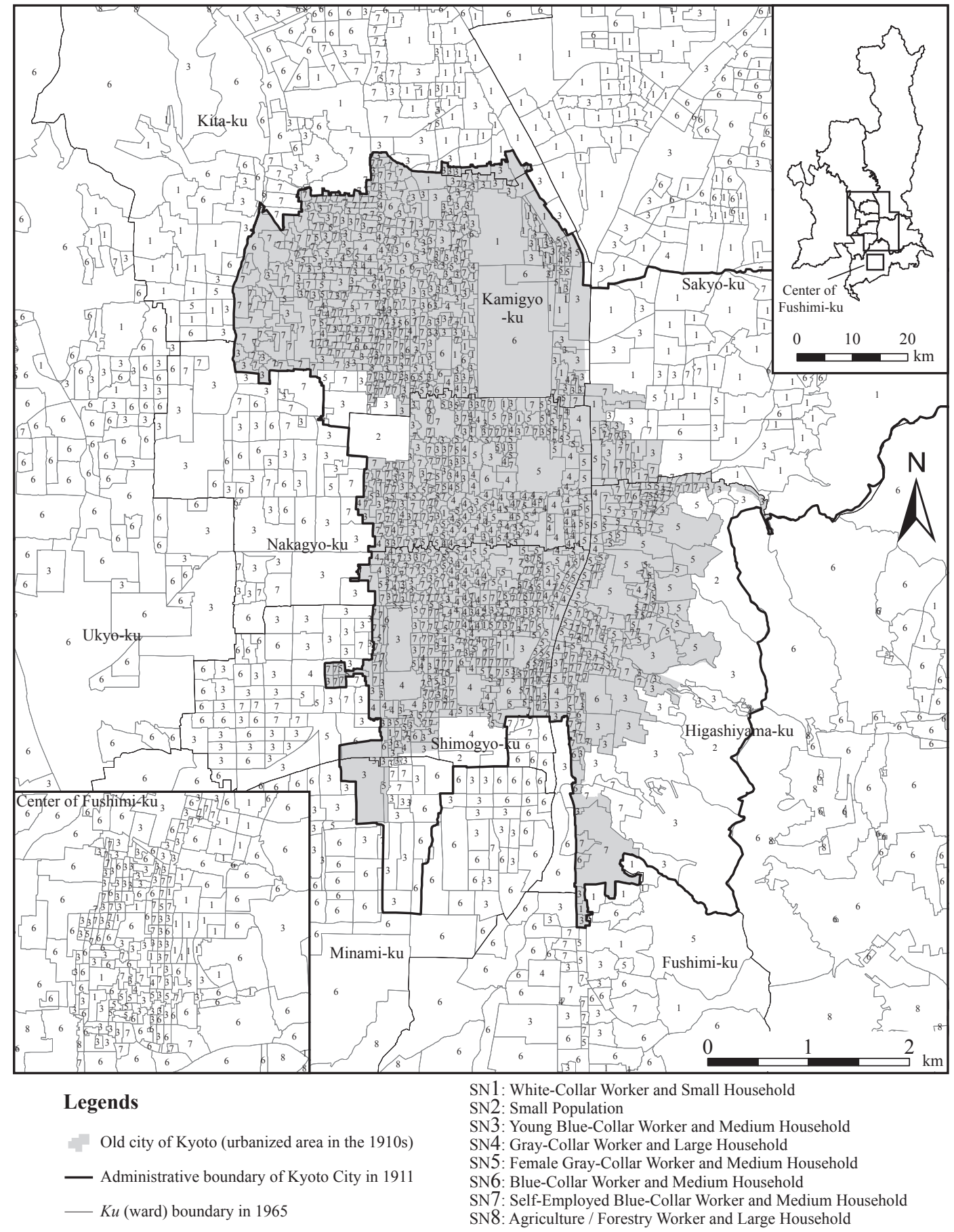

Figure 4. Distributions of neuron types in 1965

Distributions in the surrounding area are omitted. The number shows that of neuron type. 
area of Nishijin. The surrounding areas of the old city, where the SN3 type spreads, overlaps with the area rapidly urbanized from the Taisho period to the early Showa period and the area where land readjustment was implemented in the early Showa period. It seems safe to say that these surrounding areas constitute an urban area that was formed in the early Showa period since the zone restrictions of 1924 were applied only to industrial zone. On the other hand, land readjustment was also implemented in the early Showa period in the areas surrounding the eastern part of Kamigyo-ku and the southern part of Sakyo-ku, where civil servants or professional workers were dominant in 1911 and where the SN1 type spread widely at the time of 1965. The SN1 type spreads not only in the northeastern part of the urban area, but also on the north and the northwestern part of the urban area, corresponding well to the suburban residential area sold before World War 36 it.

Fushimi-ku, which was incorporated into Kyoto City in 1931, forms a structure different from that of the urban area of Kyoto centering on the old city of 1911. Along Otesuji, a commercial center of Fushimi-ku, the SN5 type spreads around the two districts that belong to the SN4 type, although they are not so concentrated as to be called accumulations. Assuming that these districts are the center of Fushimi-ku, an accumulation of the SN1 type is found toward the north and further in that direction is some accumulation in Fukakusa. In terms of the residential structure, Fushimi seems to form a city, although small in scale, since just like Kyoto's urban area, there can be found the SN3 and SN7 types between the SN6 type, which also has a rural nature and the center of Fushimi.

From these findings, the residential structure of 1965 can be summarized as follows. While inheriting the residential structure of 1911, the residential area of the blue-collar group spreads mainly in the southwestern suburbs and that of the white-collar group in the northeastern suburbs, maintaining their sectoral positioning, as a result of land readjustment projects, the development of suburban residential areas and the zone restrictions under city planning projects. Regarding household size, while the hokonin system seems to have become difficult to maintain, the concentric structure whereby the closer to the inner-city area, the larger the household, is still maintained. In the region between the residential area of the blue-collar group centering on the southwestern suburbs and the rural area that was incorporated into the Kyoto city area, a residential area under urbanization, where a mix of agriculture and industry predominates, is formed. It was also found that there was a sector-type, small-scale residential structure in Fushimi-ku, which had been newly incorporated into the Kyoto city area.

\section{(3) Residential Structure in 2005}

Lastly, SOM was applied to the residential characteristics of 2005 and eight neuron types were obtained as in 1965. Each neuron type was named in the same manner as other time points according to major occupation and household size, while giving due consideration to the characteristics of each neuron type (Table 4). The neuron type HN1 was named "White-Collar Worker and Medium Household," HN2 "Blue-Collar Worker and Medium Household H," HN3 "Agriculture / Forestry Worker and Large Household H," HN4 "White-Collar / Gray-Collar Worker and Small Household," HN5 "Middle-Aged / Senior White-Collar Worker and Medium Household," HN6 "Small Population A," HN7 "Female Gray-Collar Worker and Medium Household" and HN8 "Small Population B."

As of 2005, the city area expanded to $827.9 \mathrm{~km}^{2}$ due to the incorporation of Keihoku-cho in April 2005, while the population was $1,474,811$, an increase of approximately 100,000 compared 
Table 4. Characteristics of neuron types in 2005 (average values of neurons' weights).

\begin{tabular}{c|c|r|r|r|r|r|r|r|r}
\hline No & Variable name & \multicolumn{1}{c}{ HN1 } & \multicolumn{1}{c|}{ HN2 } & \multicolumn{1}{c}{ HN3 } & \multicolumn{1}{c}{ HN4 } & \multicolumn{1}{c}{ HN5 } & \multicolumn{1}{l}{ HN6 } & \multicolumn{1}{c}{ HN7 } & \multicolumn{1}{c}{ HN8 } \\
\hline 1 & POPDENS & 0.36 & 0.19 & -1.01 & 0.37 & -0.37 & -0.55 & -0.22 & -1.23 \\
2 & MALE & 0.29 & 0.34 & 0.34 & 0.29 & 0.16 & -1.05 & 0.03 & -2.91 \\
3 & FAMALE & 0.23 & 0.19 & 0.18 & 0.22 & 0.33 & -0.88 & 0.46 & -2.95 \\
4 & HHSIZE & 0.12 & 0.12 & 0.37 & -0.21 & 0.09 & -0.38 & 0.13 & -1.03 \\
5 & AGE00_14 & 1.14 & 0.30 & 0.14 & -0.42 & -0.14 & -0.84 & -0.54 & -1.46 \\
6 & AGE15_24 & -0.14 & -0.16 & -0.25 & 1.43 & 0.07 & 0.20 & -0.33 & -1.23 \\
7 & AGE25_29 & 0.31 & -0.11 & -0.42 & 0.84 & -0.16 & -0.66 & -0.20 & -1.37 \\
8 & AGE30_39 & 1.10 & 0.17 & -0.32 & 0.01 & -0.26 & -0.96 & -0.39 & -1.78 \\
9 & AGE40_49 & 0.70 & 0.32 & 0.50 & -0.30 & 0.13 & -0.89 & -0.15 & -1.91 \\
10 & AGE50_59 & -0.23 & 0.44 & 0.26 & -0.30 & 0.37 & -0.81 & 0.37 & -1.93 \\
11 & AGE60_69 & -0.52 & 0.42 & 0.25 & -0.35 & 0.41 & -0.71 & 0.48 & -1.74 \\
12 & AGE70_ & -0.61 & -0.02 & 0.77 & -0.23 & 0.45 & -0.19 & 1.24 & -1.35 \\
13 & OCCPOP & 0.44 & 0.28 & 0.25 & 0.03 & 0.03 & -1.20 & 0.17 & -2.73 \\
14 & EMP & 0.53 & 0.33 & -0.21 & 0.39 & 0.32 & -0.87 & -0.28 & -2.85 \\
15 & SELFEMP & -0.34 & 0.02 & 0.92 & -0.16 & 0.05 & -0.62 & 1.13 & -1.58 \\
16 & AGRW & -0.14 & -0.01 & 3.68 & -0.20 & -0.15 & -0.21 & -0.20 & -0.27 \\
17 & BLUEW & -0.04 & 0.74 & 0.37 & -0.26 & -0.36 & -0.85 & -0.10 & -1.68 \\
18 & GRAYW & -0.05 & -0.02 & -0.54 & 0.46 & -0.10 & -0.62 & 0.82 & -2.03 \\
19 & WHITW & 0.62 & -0.14 & -0.47 & 0.32 & 0.93 & -0.41 & -0.12 & -2.02 \\
\hline
\end{tabular}

to 1965. During the four decades, the relocation of factories and some universities from the city center to the surrounding areas accelerated until the 19703 s and the population in the city center of Shimogyo-ku was rapidly aging in the 1980s. However, population aging slowed down in Nakagyo-ku and Kamigyo-ku, thanks to the inflow of young people, such as university students.

According to the spatial distribution of each neuron type of 2005, the distribution range of the HN3 type, where workers in agriculture and forestry predominate, reduced along with further expansion of the urban area. While the HN3 type has similar characteristics to the SN8 type of 1965, ratios of aged persons increased and ratio of population under 15 decreased. Since it became difficult to identify the old city area of 1911 through the spatial distributions of other neuron types (Figure 5), the degree of spatial autocorrelation of each neuron type of the three time points was measured by targeting only the old city area of 1911 (Table 5). The standardized join-count statistics (hereinafter called $\mathrm{Z}-\mathrm{JC}$ ) ) was used to measure the spatial autocorrelation of the nominal scale of the types. By using $\mathrm{Z}-\mathrm{JC}$, the degree of spatial accumulation can be obtained. The larger the value of $\mathrm{Z}^{-} \mathrm{JC}$, the stronger the positive spatial autocorrelation or spatial crosscorrelation. If there is a strong positive spatial autocorrelation, then certain neuron types spread on a plane. If there is a strong positive spatial cross-correlation, two types form a spatial group, while showing a mixed distribution. Since each time point has a different number of districts, their Z-JC values cannot be compared directly. Nevertheless, while in 1911, most neuron types showed a strong autocorrelation, in 1965, the SN1, SN4 and SN5 types showed a strong positive autocorrelation and the remaining neuron types did not show any strong correlation. In 2005, since there were slightly more districts than in 1911, that is, more neighboring combinations, if the degree of spatial autocorrelation is about the same, then the Z-JC of 2005 should become greater. However, the combination with a Z-JC value of over 10 was found only in the HN8 type, which means that the spatial autocorrelation or cross-correlation in 2005 was not as strong as that in 1911. This reduction in the size of spatial cluster of neuron types in the old city of 1911 suggests that the distribution of each neuron type is becoming mosaic in the old city area. 


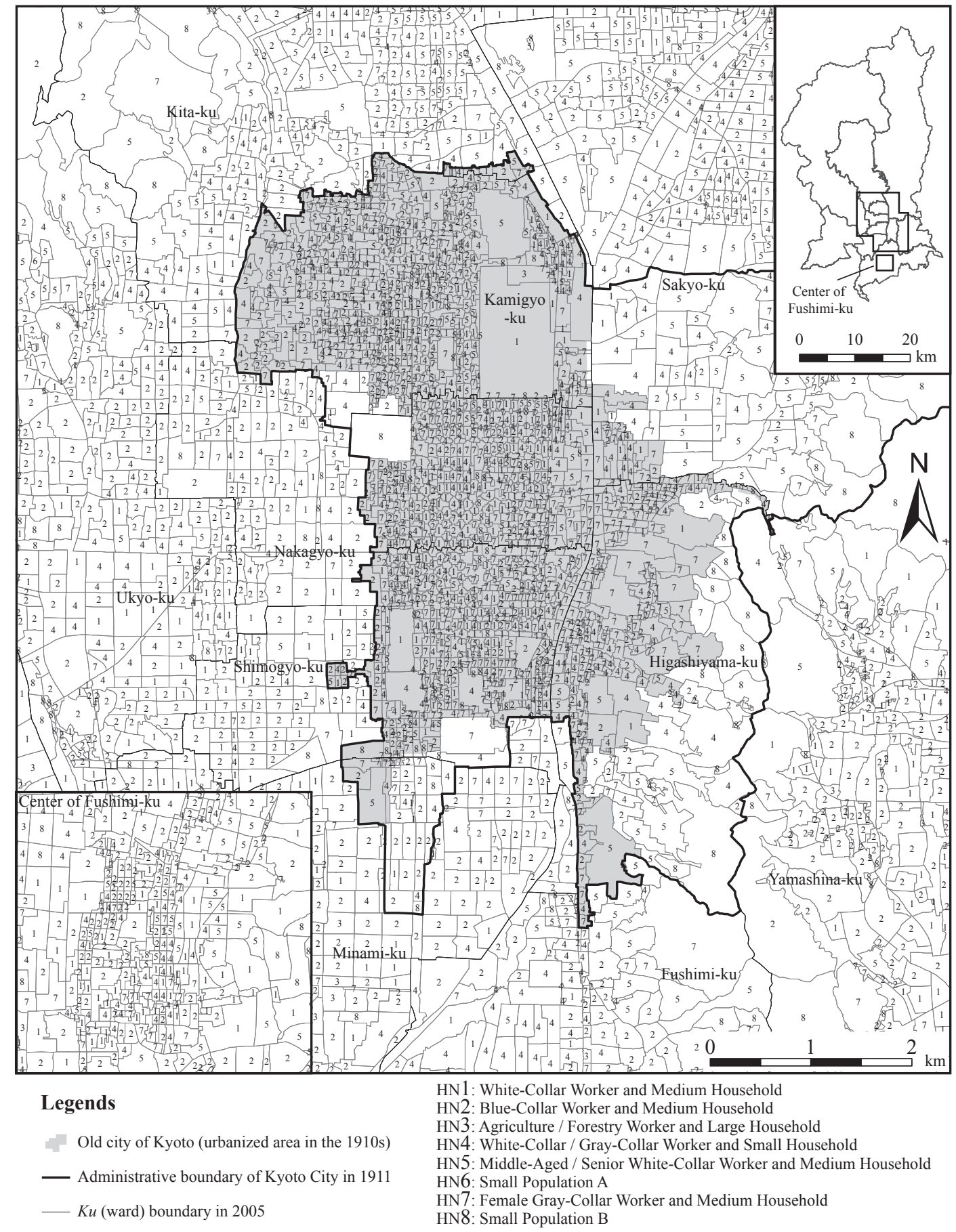

Figure 5. Distributions of neuron types in 2005.

Distributions in the surrounding area are omitted. The number shows that of neuron type. 
Table 5. Standardized join-count statistics in the administrative area of Kyoto City in 1911.

\begin{tabular}{|c|c|c|c|c|c|c|c|c|c|}
\hline & MN1 & MN2 & MN3 & MN4 & MN5 & MN6 & MN7 & MN8 & MN9 \\
\hline MN1 & 19.2 & & & & & & & & \\
\hline MN2 & 17.3 & 16.0 & & & & & & & \\
\hline MN3 & -0.7 & -1.6 & 6.1 & & & & & & \\
\hline MN4 & -3.9 & -5.1 & -1.6 & 25.4 & & & & & \\
\hline MN5 & -7.6 & -12.4 & -0.7 & 2.8 & 15.4 & & & & \\
\hline MN6 & 2.0 & -4.6 & -1.2 & -6.0 & -5.8 & 24.8 & & & \\
\hline MN7 & -6.2 & -3.3 & -1.8 & -13.5 & -7.3 & -0.7 & 10.8 & & \\
\hline MN8 & -2.3 & -2.6 & 1.9 & -8.3 & -3.7 & -1.4 & -1.8 & 15.1 & \\
\hline \multirow[t]{2}{*}{ MN9 } & -2.2 & -1.2 & -1.0 & -3.7 & -1.1 & -0.5 & 3.8 & 11.4 & 15.6 \\
\hline & SN1 & SN2 & SN3 & SN4 & SN5 & SN6 & SN7 & & \\
\hline SN1 & 22.5 & & & & & & & & \\
\hline SN2 & 0.9 & -0.2 & & & & & & & \\
\hline SN3 & 1.9 & 3.1 & 6.1 & & & & & & \\
\hline SN4 & -4.8 & -1.3 & -4.3 & 20.7 & & & & & \\
\hline SN5 & -4.7 & -0.4 & -7.4 & 7.6 & 12.9 & & & & \\
\hline SN6 & 6.4 & 0.1 & 3.9 & -1.2 & -2.5 & 3.1 & & & \\
\hline \multirow[t]{2}{*}{ SN7 } & -10.1 & -1.8 & -3.6 & -10.4 & -5.5 & -1.7 & 9.1 & & \\
\hline & HN1 & HN2 & HN3 & HN4 & HN5 & HN7 & HN8 & & \\
\hline HN1 & 5.1 & & & & & & & & \\
\hline HN2 & -2.2 & 6.9 & & & & & & & \\
\hline HN3 & 1.5 & -1.2 & - & & & & & & \\
\hline HN4 & 3.1 & -2.6 & 1.2 & 4.0 & & & & & \\
\hline HN5 & -0.1 & -0.6 & -0.7 & -0.8 & 7.7 & & & & \\
\hline HN7 & -3.9 & -4.5 & 0.9 & -1.8 & -2.1 & 8.1 & & & \\
\hline HN8 & -1.2 & -5.3 & 1.6 & -3.9 & -0.3 & -4.5 & 13.8 & & \\
\hline
\end{tabular}

With this point in mind, the distributions of neuron types centering on the old city are reviewed below. The HN7 type, for which characteristics resemble those of the SN5 type of 1965, shows a distribution centering on Higashiyama-ku. While it spreads centering on the old city area at the time of 1911 and the center of Fushimi, it could not be found in the city center, where the gray-collar group and large households used to predominate. In the center areas that existed in Kamigyo-ku, Nakagyo-ku and Shimogyo-ku, the HN1, HN4 and HN8 types are found. It seems that due to a relative increase of white-collar workers and the hollowing out of the population brought by the expansion of the business district, the conditions that the gray-collar group was relatively superior to other groups have collapsed. The population of 2005 in the old city area of 1911 was 259,861 , a decrease to about $56 \%$ of the population of 1965 . In the surrounding areas, on the other hand, white-collar-dominant three neuron types, namely, HN1, HN4 and HN5, still predominate in the northeastern suburbs, where white-collar workers used to be prominent. There is a tendency that the HN4 and HN5 types spread on the city center side and the HN1 type spread outside the other two types. On the other hand, while the HN2 type still predominates in the southeastern suburbs and Yamashina-ku, where the blue-collardominant neuron types used to concentrate, large accumulations of the HN1 and HN4 types are formed in the southern part of Ukyo-ku and the northern part of Fushimi-ku. In each of these areas, universities were established around 1960, which made them assume a nature similar to the HN4 type, where the percentage of 15-24 year olds centering on students is high. When the 


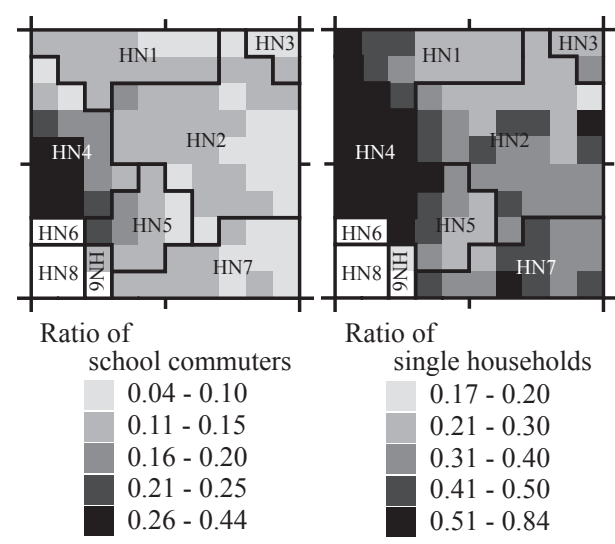

Figure 6. Average values of ratio of school commuters and ratio of single households $s$ by neurons in 2005 .

ratio of single-person households calculated by 100 neurons and the average ratio of school commuters over the age of 15 were put on the 2005 SOM map, both values were found to be high among the types that belong to the HN4 type (Figure 6). This result shows that these areas have come to assume the residential characteristics exclusive to single students due to the establishment of universities. Yamashina-ku has also become dotted with the white-collar-dominant neuron types centering on the HN1 type. The reason why the HN8 type spreads widely among the mountain areas is because the spatial unit has become smaller than that of 1965 .

Thus, compared with 1965, the distinction between the city center and the surrounding areas in the residential structure of 2005 has become obscure in terms of the distribution of neuron types and the household size. One of the reasons for this is that the residential structure of the late Edo period, which had been maintained to some extent up to 1965, was lost due to the declining population brought by the expansion of the business area, the rapid aging of the population and the inflow of students in the inner-city area. For example, in terms of the household size, the small household-dominant HN4 type has emerged in the city center and its surrounding areas, reversing the conventional structure. Moreover, in the surrounding areas, the white-collar-dominant neuron types have scattered, while forming large clusters, in the southwestern suburbs where the blue-collar group used to predominate, which suggests that the relative status of the blue-collar group in the residential characteristics has declined and that the white-collar group has expanded. In addition, the small population neuron type, which used to exist only in the mountain areas or in the districts where large-scale facilities were built, has also emerged in the city center along with a decline in the population.

\section{Conclusion}

In the above, the residential structure of Kyoto City at three time points of 1911, 1965 and 2005 over 94 years was discussed. While care should be taken in respect to changes in the spatial unit and the expansion of the administrative area when reviewing the data, the following three points should be noted concerning changes in the residential structure.

The first point is that the white-collar group also emerged in the southwestern suburbs, while the occupation-based sectoral structure comprising the center of Kamigyo-ku, Shimogyo-ku and Fushimi-ku, the blue-collar group in the western or southwestern area and the white-collar group in the northeastern area centering on the old city area of 1911 still existed at the time of 1965 and was mostly maintained in 2005. The above areas have universities and feature many students. The active construction of apartment buildings for students in these areas seems to have also prompted the inflow of young white-collar people. This encroachment by the whitecollar group can be characterized not as sectoral, but as aggregatory centering on universities, railroad stations and the area developed for housing, suggesting that the sectoral residential 
demarcation based on occupation will gradually disappear.

The second point is that the concentric pattern of household size has changed. The tendency that the closer to the city center, the larger the household, which had continued from the late Edo period to 1965, changed in 2005 to a condition where the household size was not very large, even in the city center. In the city center, there was a large-scale inflow of an age group corresponding to university students in the 1980s in Kamigyo-ku and Nakagyo-k2 ${ }^{42}$. It seems that the inflow of the group mainly of single persons was due to a change in land use from radically declining textile-related factories and offices to apartment buildings.

The third point is that although the distribution pattern of neuron types in 1965 virtually maintained that of 1911 and showed a clear image of the old city area, the pattern completely disappeared in 2005. The Z-JC values of 1965 and 2005 in the city area of 1911 show that the mosaic of spatial distribution of each neuron type in the old city area has accelerated during the 40-year period from 1965 to 2005. This is because as mentioned in the second point, various changes in residential characteristics in the city center that occupied most of the old city area were not uniform or spread widely on a plane.

The residential structure of Kyoto City from 1911 to 1965 maintained the structure in the city center that had been inherited since the late Edo period and the structure newly added after the Meiji period, while expanding a similar structure into the surrounding areas. In 2005, the residential structure made a drastic change centering on the aforementioned three changes. While there was a tendency that the sectoral structure based on occupation was encroached upon by the white-collar group in an agglomerative manner in the blue-collar-dominant surrounding areas, the mosaic of residential characteristics accelerated in the city center. In light of the residential structure of 2005, it seems that it will become more difficult to apply the traditional framework of intra-urban structure, such as a concentric structure comprising an city center and its surrounding areas and a sectoral structure based on occupation, at least to the residential structure of Kyoto City.

In this paper, discussion was carried out with reference to only three time points of 1911, 1965 and 2005. However, since a drastic change in the residential structure of Kyoto City took place during the period from 1965 to 2005, elucidation of detailed changes in the residential structure during this period is expected. Moreover, due to limited materials, the residential characteristics of the surrounding towns and villages of Kyoto City in 1911 were not included or analyzed in this paper, which made the examination of changes in the expanded city area incomplete. This should be examined further in the future.

\section{Acknowledgements}

The work has supported by the Grant-in-Aid for Scientific Research (19-8458) by the JSPS and the Global COE program (Digital Humanities Center for Japanese Cultures and Arts) by the MEXT. This paper was presented at the 14th International Conference of Historical Geographers, Kyoto University, at Japan, 23-27 August 2009.

\footnotetext{
Notes

1) Ueno, K., 'A Review on the Study of Urban Residential Structures : on the Relationships between Factorial Ecology and Urban Geography', Geographical Review of Japan, 55, 1982, pp. 715-734. (JE)

2) Mizuuchi, T., 'Formation and Development of the Inner City in Osaka during the Japanese Industrialisation', The Human Geography (Jimbun Chiri), 34, 1982, pp. 385-409. (JE)

3) Ueno, K., 'Residential Structure of Tokyo during the Taisho Era (1920) : A Factorial Ecological Study of the Socio-
} 
Economic Characteristics of Residential Population', The Human Geography (Jimbun Chiri), 33, 1981, pp. 385-404. (JE)

4) Ueno, K., 'The Residential Structure of Tokyo in the Post World War II Period of Showa (1970) ', Tsukuba Studies in Human Geography, 20, 1996, pp. 241-254. (JE)

5) Tomita, K., 'Sandaitoshiken ni okeru chiikihennyou' (Sugiura, Y., ed., Kukan no Keizai Chiri, Asakura Publishing, 2004), pp. 80-105. (J)

6) Tanaka, M., Okata, J. and Koizumi, H., 'The Analysis of Population-decline in the Tokyo "KU" Area after 1980's', Urban Housing Sciences, 26, 1999, pp. 127-135. (JE)

7) Kagawa, T., 'Kyoto-shi ni okeru jinko koureika no shosou', Geographical Sciences, 46, 1991, pp. 158-163. (J)

8) Miyazawa, H. and Abe, T., 'Recovery and Changes in the Socioeconomic Composition of Population in the Central Area of Tokyo during the Period from 1995 to 2000 : Analysis of Small-area Census Data', Geographical Review of Japan, 78, 2005, pp. 893-912. (JE)

9) op. cit., footnote 3 ).

10) Tanabe, K., 'A Geographical Study on the Reestablishment of the Destroyed City, Sendai', Geographical Review of Japan, 22, 1949, pp. 264-273. (J)

11) (1) Kyoto City Office, Kyoto-shi rinji jinko chosa youkei hyo dai 1 hen, Kyoto City Office, 1912. (J), (2) Kyoto City Office, Kyoto-shi rinji jinko chosa youkei hyo dai 2 hen, Kyoto City Office, 1913. (J)

12) Hamano, K., Kinsei Kyoto no rekishi jinkogaku teki kenkyu: Toshi chonin no shakai kouzo wo yomu, Keio University Press, 2007. (J)

13) Ito, Y., 'Toshi keiei to Kyoto-shi no kaizo jigyo no keisei : 1895-1907' (Ito, Y. ed., Kindai Kyoto no kaizo: Toshi keiei no kizgen 1850-1918, Minerva Publishing, 2006), pp. 31-82. (J)

14) op. cit., footnote 11) (1).

15) op. cit., footnote 11) (1).

16) Center for Statistics Analysis of Kyoto City Office, Kyoto-shi no jinko, Kyoto City Office, 1967. (J)

17) Center for Statistics Analysis of Kyoto City Office, Kyoto-shi no chobetsu jinko, Kyoto City Office, 1966. (J)

18) op. cit., footnote 11) (2).

19) Kirimura, T., 'An Application of Self-organizing Maps to the Research of Residential Structure : Does the Dimension of Economic Status Exist in Kyoto in 1970 ?', The Journal of Ritsumeikan Geographical Society, 18, 2006, pp. 5567. (J)

20) Kohonen, T., 'The Self-organizing Map', Neurocomputing, 21, 1998, pp. 1-6.

21) Nakaya, T., 'Neural network' (Sugiura, Y., ed., Chiri kukan bunseki, Asakura Publishing, 2003), pp. 175-195. (J)

22) op. cit., footnote 19).

23) op. cit., footnote 21).

24) Openshaw, S., Blake, M. and Wymer, C., 'Using neurocomputing methods to classify Britain's residential areas' (Fisher, P., ed., Innovations in GIS 2, Taylor \& Francis, 1995), pp. 97-111.

25) Kirimura, T., 'Geographical Clustering of Small Areas : Handling Outliers and Spatial Smoothing', Theory and Applications of GIS, 15, 2007, pp. 33-44. (JE)

26) Osuga, T., 'Kyoto-shi no shigaichi keisei no hensen to tokushoku' (Meeting of Modern Urban Policy) 2005.

27) Kato, M., Kyo no kagai monogatari, Kadokawa Gakugei Shuppan Publishing, 2009. (J)

28) op. cit., footnote 12).

29) op. cit., footnote 12).

30) op. cit., footnote 3).

31) op. cit., footnote 26).

32) (1) Fujimoto, T., 'Kyoto bonchi ni okeru nogyo shuraku', The Human Geography (Jimbun Chiri), 13, 1961, pp. 133-138. (J), (2) Kubota, T., 'Kojo tekichi chosa yori mita Keihan kan no nairiku kogyo ni tsuite', The Human Geography (Jimbun Chiri) , 13, 1961, pp. 335-346. (J), (3) Tsuji, F., 'Formation of Residential Areas and Flood Disaster in the West-southern Lowlands of Kyoto', The Human Geography (Jimbun Chiri), 20, 1968, pp. 616-644. (JE)

33) op. cit., footnote 32) (1).

34) Matsui, K., 'The Structure of Nishijin Industrial Region; Study of the Small-scale Wearing Industry in Kyoto Metropolitan Area, The Human Geography (Jimbun Chiri), 31, 1979, pp. 117-136. (JE)

35) Mizuuchi, T., Kato, M. and Oshiro, N., Modan toshi no keifu: Chizu kara yomitoku shakai to kukan, Nakanishiya Shuppan, 2008. (J)

36) op. cit., footnote 26).

37) Kiji, S., 'The Market Area of the Shopping Streets in Kyoto City', The Human Geography (Jimbun Chiri), 9, 1957, pp. 97-108. (JE)

38) op. cit., footnote 34), (1) Kawashima, K., 'How did the Private and Imperial Universities Get their Own Space in Kyoto ?: A Geographical Essay on the Change in Land Use after the Meiji Restoration', The Journal of Ritsumeikan 
Geographical Society, 17, 2005, pp. 129-139. (J), (2) Kaido, S., 'The Characteristics of the Printing Industry in Kyoto City : From the Results of Prefecture-wise Analysis', The Human Geography (Jimbun Chiri), 29, 1977, pp. 88-102. (J).

39) op. cit., footnote 7).

40) Cliff, A. D. and Ord, J. K., Spatial Processes: Models and Applications, Pion, 1981.

41) op. cit., footnote 25).

42) op. cit., footnote 7).

43) (1) Fujitsuka, Y., 'Burgeon of Gentrification in Nishijin, Kyoto', The Human Geography (Jimbun Chiri), 44, 1992, pp. 495-506. (JE), (2) Koga, S., 'Spatial Change in the Business District of the Central Area of Kyoto due to Office Location Changes during the Second Half of the 1990s', Geographical Review of Japan, 80, 2007, pp. 138-151. (JE)

\title{
Changes in Residential Structure in 20th-Century Kyoto City
}

\author{
KIRIMURA Takashi \\ Graduate Student Graduate School of Letters, Ritsumeikan University JSPS Research Fellow
}

The purpose of this paper is to elucidate long-term changes in residential structure in 20thcentury Kyoto City. My research focuses on the three time points of 1911, 1965, and 2005 because of the availability of small area statistics on residents. These small areas under the administration of the Kyoto City Government in the targeted time points are classified into types based on residential characteristics. Comparisons of spatial distributions of the types in each time point reveal the following three characteristics : First of all, while each time point shows sectoral spatial distributions of types in the occupation, the distributions in 2005 do not show this tendency so clearly as before. Second, as of 1965 , one can easily recognize a concentric pattern of household size, that is, the closer to the city center, the larger the household. Yet, that changed in 2005, going in the opposite direction. Finally, in the city center, while spatial distributions of the types show clear patterns in 1911 and 1965, the types in 2005 come to form a mosaic pattern. Until 1965, the residential structures had remained unchanged since the late Edo period. However, they changed drastically during the four decades after 1965. In 2005, typical models of intraurban structures such as sectoral and concentric ones have become irrelevant when one interprets Kyoto's residential structures.

Keywords : residential structure, intra-urban structure model, Kyoto City, Self-Organizing Map (SOM) 